\title{
A Highly Efficient and Green Catalytic Synthesis of 3,4-dihydro-pyrimidin-2-(1 H)-ones (Thiones) Using 3-sulfonic Acid-1-imidazolopyridinium Hydrogen Sulfate under Solvent-free Conditions
}

\author{
Mohammad Bakherad, ${ }^{1,}{ }^{*}$ Mohaddeseh Javanmardi, ${ }^{1}$ Raheleh Doost ${ }^{1}$, Reza Tayebee $^{2}$
}

\footnotetext{
${ }^{1}$ School of Chemistry, Shahrood University of Technology, Shahrood, 3619995161, Iran

2 Department of Chemistry, Hakim Sabzevari University, Sabzevar, 9617976487, Iran

* Corresponding author's e-mail address: m.bakherad@yahoo.com
}

\begin{abstract}
The ionic liquid catalyst 3-sulfonic acid-1-imidazolopyridinium hydrogen sulfate, [Simp] $\mathrm{HSO}_{4}$ was found to be a highly active and green catalyst for the synthesis of 3,4-dihydropyrimidin-2(1H)-ones (thiones) under the solvent-free conditions. Avoiding organic solvents during the chemical reactions leading to an economic approach is effective. The reactions are characterized by high efficiency, short reaction time, high product yield, simple experimental procedure, availability of catalyst, and environmentally-friendly reaction conditions.
\end{abstract}

Keywords: Biginelli reaction, [Simp] $\mathrm{HSO}_{4}$ catalyst; 3,4-dihydropyrimidin-2(1H)-ones (thiones), solvent-free.

\section{INTRODUCTION AND RESULTS}

3 4-DIHYDROPYRIMIDIN-2(1H)-ONES (DHPMs) belong to an important class of heterocyclic compounds, have attracted much interest due to their pharmacological and biological properties such as the anti-hypertensive, calcium channel blocking, anti-tumor, anti-bacterial, and antiinflammatory activities. ${ }^{[1,2]}$ The pyrimidine derivative MKC442 is already in clinical trials and similar compounds are expected to inhibit the HIV virus. ${ }^{[3]}$ Moreover, several marine alkaloids whose molecular structures contain the 3,4-dihydropyrimidin-2(1H)-ones core, also exhibit interesting biological activities. ${ }^{[4]}$ Therefore, the synthesis of these compounds is still of great importance.

The earliest method for the synthesis of 3,4dihydropyrimidin-2 $(1 \mathrm{H})$-ones reported by Biginelli in 1893 , involved the one-pot condensation of an aldehyde, an $\alpha, \beta$ ketoester, and urea under strongly acidic conditions with low product yields (often $20-50 \%$ ) $^{[5]}$ Although there are different methods for the synthesis of Biginelli's products based on the use of strong protic acids such as $\mathrm{HCl},{ }^{[6]}$ Lewis acids like $\mathrm{BF}_{3} . \mathrm{OEt}_{2}{ }^{[7]}$ zeolites, ${ }^{[8]}$ and metal trifles ${ }^{[9]}$ a variety of other conditions such as the ultrasonic, ${ }^{[10]}$ and microwave-assisted ${ }^{[11]}$ also have been reported in the literature.

However, many of these methods suffer from drawbacks such as the use of expensive reagents, strongly acidic conditions, long reaction times, and use of expensive and poisonous solvents. Therefore, the introduction of more efficient and milder methods accompanied with higher product yields is in demand. In recent years, with increase in the environmental consciousness in chemical research and industry, the solvent-free Biginelli reaction has attracted much attention and received good results recently. ${ }^{[12,13]}$

lonic liquids have fascinated much interest in the past few decades because of their unique properties including high thermal and chemical stability, nonvolatility, non-flammability, favorable solvation behavior, and wide range of liquid-state temperatures. ${ }^{[14]}$ These compounds have been extensively applied to the extraction and separation processes in industry. ${ }^{[15]}$ Most of the 
Table 1. Investigation of catalyst effects and temperature in the synthesis of 5-(Ethoxycarbonyl)-6-methyl-4-phenyl-3,4dihydropyrimidin-2(1H)-one under solvent-free conditions ${ }^{(a)}$

\begin{tabular}{ccccc}
\hline Entry & Catalyst amount $(\mathrm{mol} \%)$ & Temperature $\left({ }^{\circ} \mathrm{C}\right)$ & Time $(\mathrm{min})$ & Yields $(\%)^{(\mathrm{b})}$ \\
\hline 1 & 0 & 50 & 360 & 30 \\
2 & 0.5 & 50 & 45 & 80 \\
3 & 1.0 & 50 & 45 & 98 \\
4 & 2.0 & 50 & 45 & 98 \\
5 & 3.0 & 50 & 45 & 99 \\
6 & 1.0 & r.t & 360 & 40 \\
7 & 1.0 & 80 & 45 & 98 \\
\hline
\end{tabular}

(a) Reaction conditions: benzaldehyde $(1.0 \mathrm{mmol})$, ethyl acetoacetate $(1.0 \mathrm{mmol})$, urea $(1.2 \mathrm{mmol})$.

(b) Isolated yields.

Brønsted acidic ionic liquids are environmentally friendly, efficient, and simple acid catalysts, and have been used in various organic transformations. ${ }^{[16-18]}$

Now, in continuation of our works on the synthesis of heterocyclic compounds, ${ }^{[19-21]}$ we wish to report the use of the naturally-based ionic liquid 3-sulfonic acid-1imidazolopyridinium hydrogen sulfate, [Simp] $\mathrm{HSO}_{4}$, as a catalyst in the eco-friendly and practical synthesis of 3,4dihydropyrimidin-2(1H)-ones (thiones) via a one-pot, three-component condensation of aromatic aldehydes, $\alpha, \beta$-dicarbonyl compound and urea or thiourea under the solvent-free conditions (Scheme 1).

The ionic liquid 3-sulfonic acid-1-imidazolopyridinium hydrogen sulfate [Simp]HSO4 has been prepared by the reaction of chlorosulfonic acid with caffeine previously and was characterized thoroughly and has been used as a catalyst for the synthesis of $2 \mathrm{H}$-indazolo[2,1b]phthalazine-1,6,11(13H)-triones. ${ }^{[21]}$ In order to optimize the reaction conditions, the reaction of benzaldehyde $1 \mathrm{a}$, with ethyl acetoacetate $\mathbf{2} \mathbf{a}$ and urea $\mathbf{3} \mathbf{a}$ was chosen as a model reaction in the absence and presence of the [Simp] $\mathrm{HSO}_{4}$ catalyst in order to establish the effectiveness of the catalyst under the thermal solvent-free conditions (Table 1).

It was found that the product 4a was produced in a low yield after $4 \mathrm{~h}$ in the absence of the [Simp] $\mathrm{HSO}_{4}$ catalyst (Table 1, entry 1), whereas $1 \mathrm{~mol} \%$ of the catalyst led to a $98 \%$ product yield after $45 \mathrm{~min}$ (Table 1, entry 3 ). As shown in Table 1, this transformation required the presence of the catalyst, and, therefore, to optimize the reaction conditions, the above-mentioned model reaction was carried out under different reaction conditions. The yield of the corresponding 3,4-dihydropyrimidin-2(1H)-one 4a increased with increase in the catalyst concentration from 0.5 to $1.0 \mathrm{~mol} \%$ (Table 1, entries 2 and 3). Further addition of the catalyst had no noticeable effect on the product yield (Table 1, entries 4 and 5). Therefore, in all the further reactions $1.0 \mathrm{~mol} \%$ of the catalyst was used. The reaction

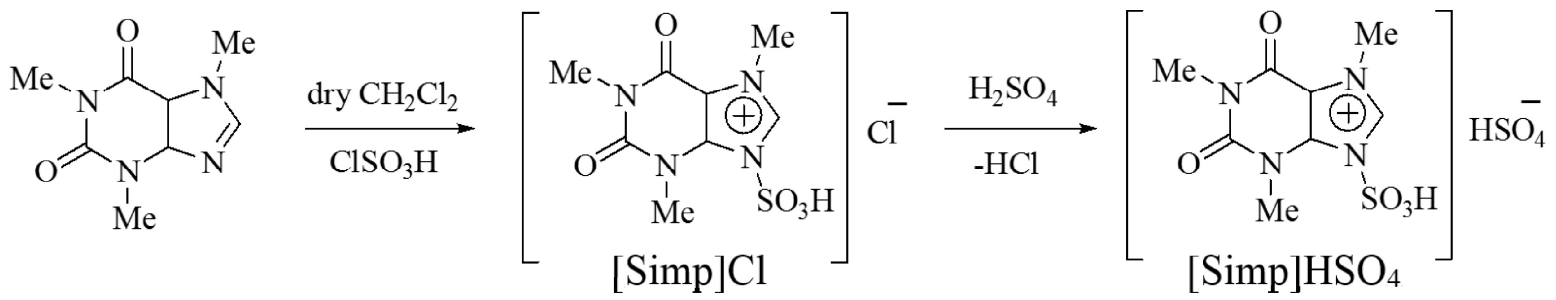<smiles>[R]C(=O)CC([CH2+])=O</smiles>

Scheme 1. 
Table 2. Solvent effect on the reaction of benzaldehyde, ethyl acetoacetate and urea catalyzed by [Simp] $\mathrm{HSO}_{4}$ (a) $^{(\mathrm{S}}$

\begin{tabular}{|c|c|c|c|}
\hline Entry & Solvent (reflux) & Time (h) & Yields $(\%)^{(b)}$ \\
\hline 1 & $\mathrm{CH}_{3} \mathrm{CN}$ & 10 & 55 \\
\hline 2 & $\mathrm{EtOH}$ & 10 & 50 \\
\hline 3 & THF & 10 & 35 \\
\hline 4 & $\mathrm{H}_{2} \mathrm{O}$ & 10 & 45 \\
\hline 5 & 1,4-Dioxan & 10 & 65 \\
\hline 6 & Tolouene & 10 & 60 \\
\hline 7 & Solvent-free & 0.75 & 98 \\
\hline
\end{tabular}

(a) Reaction conditions: benzaldehyde $(1.0 \mathrm{mmol})$, ethyl acetoacetate $(1.0 \mathrm{mmol})$, urea $(1.2 \mathrm{mmol})$.

(b) Isolated yields.

yield was susceptible to temperature changes in the presence of $1.0 \mathrm{~mol} \%$ of the catalyst under the solvent-free conditions. It was found that at room temperature, the reaction proceeded slowly, giving a low product yield (Table

Table 3. Synthesis of 3,4-dihydropyrimidin-2-(1H)-ones (thiones) $4^{(a)}$<smiles>[R]C(=O)CC([CH2+])=O</smiles>

\section{$\begin{array}{lll}1 & 2 & 3\end{array}$}

\begin{tabular}{|c|c|c|c|c|c|c|c|}
\hline Entry & $\mathrm{Ar}$ & $R$ & $x$ & Product & Time (min) & Yield (\%) & [ref.] \\
\hline 1 & $\mathrm{Ph}$ & OEt & 0 & $4 a$ & 45 & 98 & [23] \\
\hline 2 & 4-Me- $\mathrm{C}_{6} \mathrm{H}_{4}$ & OEt & 0 & $4 b$ & 50 & 80 & [24] \\
\hline 3 & 4- $\mathrm{MeO}-\mathrm{C}_{6} \mathrm{H}_{4}$ & OEt & $\mathrm{O}$ & $4 c$ & 60 & 70 & [24] \\
\hline 4 & $2,3,4-(\mathrm{MeO})_{3}-\mathrm{C}_{6} \mathrm{H}_{2}$ & OEt & 0 & $4 d$ & 20 & 50 & This work \\
\hline 5 & $4-\mathrm{Cl}_{-}-\mathrm{C}_{6} \mathrm{H}_{4}$ & OEt & $\mathrm{O}$ & $4 e$ & 30 & 60 & [24] \\
\hline 6 & $2-\mathrm{Cl}-\mathrm{C}_{6} \mathrm{H}_{4}$ & OEt & $\mathrm{O}$ & $4 f$ & 120 & 98 & [25] \\
\hline 7 & $4-\mathrm{NO}_{2}-\mathrm{C}_{6} \mathrm{H}_{4}$ & OEt & 0 & $4 \mathrm{~g}$ & 120 & 60 & [26] \\
\hline 8 & 4-CHO- $\mathrm{C}_{6} \mathrm{H}_{4}$ & OEt & 0 & $4 \mathrm{~h}$ & 30 & 97 & [27] \\
\hline 9 & $\mathrm{Ph}$ & OEt & S & $4 i$ & 30 & 36 & [26] \\
\hline 10 & 2-Me- $\mathrm{C}_{6} \mathrm{H}_{4}$ & OEt & S & $4 j$ & 60 & 98 & [28] \\
\hline 11 & 4- $\mathrm{MeO}-\mathrm{C}_{6} \mathrm{H}_{4}$ & OEt & S & $4 k$ & 30 & 92 & [26] \\
\hline 12 & $4-\mathrm{Cl}-\mathrm{C}_{6} \mathrm{H}_{4}$ & OEt & $\mathrm{s}$ & 41 & 300 & 98 & [26] \\
\hline 13 & $2-\mathrm{Cl}-\mathrm{C}_{6} \mathrm{H}_{4}$ & OEt & S & $4 m$ & 300 & 40 & [29] \\
\hline 14 & 4-CHO- $\mathrm{C}_{6} \mathrm{H}_{4}$ & OEt & S & $4 n$ & 45 & 85 & [30] \\
\hline 15 & $\mathrm{Ph}$ & Me & 0 & 40 & 40 & 55 & [23] \\
\hline 16 & 4-Me- $\mathrm{C}_{6} \mathrm{H}_{4}$ & $\mathrm{Me}$ & $\mathrm{O}$ & $4 p$ & 45 & 97 & [23] \\
\hline 17 & $2,3,4-(\mathrm{MeO})_{3}-\mathrm{C}_{6} \mathrm{H}_{2}$ & $\mathrm{Me}$ & 0 & $4 q$ & 15 & 60 & This work \\
\hline 18 & $4-\mathrm{Cl}_{-}-\mathrm{C}_{6} \mathrm{H}_{4}$ & Me & 0 & $4 r$ & 30 & 40 & [23] \\
\hline 19 & $2-\mathrm{Cl}-\mathrm{C}_{6} \mathrm{H}_{4}$ & $\mathrm{Me}$ & 0 & $4 s$ & 30 & 53 & [29] \\
\hline 20 & 4-CHO- $\mathrm{C}_{6} \mathrm{H}_{4}$ & $\mathrm{Me}$ & 0 & $4 t$ & 30 & 90 & [27] \\
\hline 21 & $\mathrm{Ph}$ & Me & S & $4 u$ & 30 & 80 & [23] \\
\hline 22 & 4-Me- $\mathrm{C}_{6} \mathrm{H}_{4}$ & Me & S & $4 v$ & 35 & 98 & [23] \\
\hline 23 & $4-\mathrm{Cl}-\mathrm{C}_{6} \mathrm{H}_{4}$ & Me & S & $4 w$ & 60 & 97 & [29] \\
\hline 24 & $2-\mathrm{Cl}-\mathrm{C}_{6} \mathrm{H}_{4}$ & Me & S & $4 x$ & 40 & 98 & [29] \\
\hline 25 & 4-CHO- $\mathrm{C}_{6} \mathrm{H}_{4}$ & $\mathrm{Me}$ & S & $4 y$ & 30 & 93 & [27] \\
\hline
\end{tabular}

(a) Reaction conditions: Aromatic aldehyde $(1.0 \mathrm{mmol}), \alpha, \beta$-dicarbonyl compound $(1.0 \mathrm{mmol})$ urea or thiourea $(1.2 \mathrm{mmol})$, catalyst $(0.01 \mathrm{mmol})$, solvent-free, at $50 \cong$. .

(b) Isolated yields. 
Table 4. Comparison of protocols for the synthesis of 3,4-dihydropyrimidin-2(1H)-one $4 a^{(a)}$

\begin{tabular}{|c|c|c|c|c|}
\hline Entry & Catalyst & Condition & Time & Yield (\%) ${ }^{\text {[ref.] }}$ \\
\hline 1 & $\mathrm{H}_{3} \mathrm{PMo}_{12} \mathrm{O}$ & $\mathrm{CH}_{3} \mathrm{CN}, 80^{\circ} \mathrm{C}$ & $1 \mathrm{~h}$ & $93^{[30]}$ \\
\hline 2 & $\mathrm{H}_{3} \mathrm{PW}_{12} \mathrm{O}_{40} / \mathrm{SiO}_{2}$ & $\mathrm{CH}_{3} \mathrm{CN}, 80^{\circ} \mathrm{C}$ & $50 \mathrm{~min}$ & $97[31]$ \\
\hline 3 & Nano- $\nu-\mathrm{Fe}_{2} \mathrm{O}_{3}-\mathrm{SO}_{3} \mathrm{H}$ & Solvent-free, $60^{\circ} \mathrm{C}$ & $3 \mathrm{~h}$ & $95^{[25]}$ \\
\hline 4 & HPVMo 11 & $\mathrm{EtOH}, 80^{\circ} \mathrm{C}$ & $6 \mathrm{~h}$ & $95^{[32]}$ \\
\hline 5 & $\mathrm{CH}_{3} \mathrm{COOH}$ & MW & $7 \mathrm{~min}$ & $84^{[33]}$ \\
\hline 6 & $\mathrm{HClO}_{4}$ & $\mathrm{MW}, 240 \mathrm{~W}$, solvent-free & $2 \min$ & $95^{[34]}$ \\
\hline 7 & [Simp]HSO4 & Solvent-free, $50^{\circ} \mathrm{C}$ & $45 \mathrm{~min}$ & $98^{\text {[a] }}$ \\
\hline
\end{tabular}

(a) Referred to the present work.

1 , entry 6). The increase in the reaction temperature up to $50 \stackrel{\circ}{\circ}$ led to an excellent yield (Table 1, entry 3 ).

To check the solvent effect on the outcome of the reaction, the above-mentioned model reaction was carried out using $1.0 \mathrm{~mol} \%$ of the catalyst in solvents such as $\mathrm{CH}_{3} \mathrm{CN}$, EtOH, THF, $\mathrm{H}_{2} \mathrm{O}, 1$,4-dioxan, and toluene under the reflux conditions (Table 2). All the screened solvents afforded low product yields after $10 \mathrm{~h}$. It is noteworthy that when THF was used as the solvent, a lower product yield was observed (Table 2, entry 3 ). The best product yield was obtained under the solvent-free conditions at $50{ }^{\circ} \mathrm{C}$ (Table 2 , entry 7 ). Thus all the reactions were performed in the presence of $1 \mathrm{~mol} \%$ of the catalyst at $50{ }^{\circ} \mathrm{C}$ under the solvent-free conditions.

To extend the scope of the reaction, and to generalize the procedure, we investigated the reactions of a series of aromatic aldehydes with urea (thiourea) and ethyl acetoacetate or acetylacetone under the optimized reaction conditions. In all the cases studied, the threecomponent reaction proceeded smoothly to give the corresponding 3,4-dihydropyrimidin-2(1H)-ones (thiones) with satisfactory yields. The aromatic aldehydes carrying either electron-donating or electron- withdrawing substituents at the ortho, meta, and para positions afforded high yields of 3,4-dihydropyrimido-2(1H)-ones. An important feature of this procedure is the survival of a variety of functional groups such as nitro, chloro, methyl, and methoxy under the reaction conditions. In addition thiourea reacted under similar reaction conditions to form the corresponding 3,4-dihydropyrimido-2(1H)-thiones in good to excellent yields (Table 3 ).

In order to show the merit of this catalytic method, the results obtained for the reactions of benzaldehyde, ethyl acetoacetate, urea, (Table 4) were compared with those obtained for some other catalysts used for the same reactions. Most of the listed methodologies suffer from some limitations such as prolonged reaction times, elevated temperatures, and use of hazardous materials. For example, preparation of 3,4-dihydropyrimido-2(1H)-one $\mathbf{4 a}$ was carried out in the hazardous $\mathrm{CH}_{3} \mathrm{CN}$ (Table 4, entries 1 , and 2). Additionally, the present protocol was effective in the synthesis of compound $4 \mathrm{a}$ in a reaction time of $45 \mathrm{~min}$. The same transformation required 3-6 $\mathrm{h}$ for completion by using other catalysts. As it is evident in the results tabulated in Table 4, [Simp]HSO4 is more efficient than the other catalysts.

In conclusion, in this work, a catalytic amount of [Simp] $\mathrm{HSO}_{4}$ was used very effectively, for the first time, for a solvent-free, high yield, and rapid synthesis of 3,4dihydropyrimido-2 $(1 H)$-ones (thiones). This method is very general and tolerates different types of functional groups. Based on our studies, this method offers several advantages including the cheapest materials, mild reaction conditions, high product yield, shorter reaction time, very facile purification, and environmentally- friendly procedure.

\section{EXPERIMENTAL}

Melting points were recorded on an electrothermal type 9100 melting point apparatus. IR spectra were obtained on a 4300 Shimadzu spectrometer as $\mathrm{KBr}$ disk. ${ }^{1} \mathrm{H}-\mathrm{NMR}$ spectra were recorded on a Bruker BRX 400 AVANCE spectrometer. Elemental analysis results were obtained using a Thermo Finnigan Flash EA microanalyser.

\section{Preparation of 3-sulfonic Acid-1- imidazolopyridinium Hydrogen Sulfate [Simp] $\mathrm{HSO}_{4}$}

To a $100 \mathrm{~mL}$ round-bottomed flask containing caffeine $(0.9710 \mathrm{~g}, 5 \mathrm{mmol})$ in dry $\mathrm{CH}_{2} \mathrm{Cl}_{2}(50 \mathrm{~mL})$, was added chlorosulfonic acid $(0.59 \mathrm{~g}, 5.1 \mathrm{mmol})$ drop-wise over a period of $20 \mathrm{~min}$ at room temperature. After the addition was completed, the reaction mixture was stirred for $12 \mathrm{~h}$. The residue was washed with dry $\mathrm{CH}_{2} \mathrm{Cl}_{2}(3 \times 50 \mathrm{~mL})$ and dried to give 3-sulfonic acid imidazolopyridinium chloride ([Simp]Cl) as a viscous pale yellow oil. Then sulfuric acid ( $98 \%)(0.49 \mathrm{~g}$, $5 \mathrm{mmol}$ ) was added drop-wise to [Simp]Cl ( $1.55 \mathrm{~g}, 5 \mathrm{mmol})$ over a period of $5 \mathrm{~min}$ at room temperature to remove the $\mathrm{HCl}$ produced during the reaction. Finally, the resulting mixture was stirred for $24 \mathrm{~h}$ at $60{ }^{\circ} \mathrm{C}$ under the aerobic conditions to give [Simp] $\mathrm{HSO}_{4}$ as a viscous yellow oil.[22] 


\section{General Procedure for the Synthesis of 3,4-dihydropyrimidin-2(1H)-ones (thiones)}

A mixture of an aromatic aldehyde $(1.0 \mathrm{mmol})$, ethyl acetoacetate $(1.0 \mathrm{mmol}, 0.13 \mathrm{~mL})$ or acetylacetone $(1.0$ $\mathrm{mmol}, 0.1 \mathrm{~mL})$, urea $(1.2 \mathrm{mmol}, 0.072 \mathrm{~g})$, or thiourea (1.2 $\mathrm{mmol}, 0.086 \mathrm{~g})$, and $[\mathrm{Simp}] \mathrm{HSO}_{4}(0.01 \mathrm{mmol}, 0.004 \mathrm{~g})$ was heated at $50{ }^{\circ} \mathrm{C}$ for an appropriate time (Table 3). After completion of the reaction, the residue was washed with water for several times. The filtrate was recrystallized from ethanol to afford the pure product.

Ethyl 1,2,3,4-tetrahydri-6-methyl-oxo-4phenylpyrimidine-5-carboxylate (4a):

White solid, (98\%); m.p. $=205-207$; ${ }^{1 \mathrm{H}} \mathrm{NMR}(400 \mathrm{MHz}$, DMSO- $\left.d_{6}\right) \delta / p p m: 1.01\left(\mathrm{t}, J=7.2 \mathrm{~Hz}, 3 \mathrm{H}, \mathrm{CH}_{3} \mathrm{CH}_{2} \mathrm{O}\right), 2.25$ $\left(\mathrm{s}, 3 \mathrm{H}, \mathrm{CH}_{3}\right), 3.98\left(\mathrm{q}, J=7.2 \mathrm{~Hz}, 2 \mathrm{H}, \mathrm{CH}_{2}\right), 5.14(\mathrm{~s}, 1 \mathrm{H}, \mathrm{C} 4 \mathrm{H})$, 7.24-7.40 (m, 3H, C'3-H, C'4-H, C'5-H), 7.33-7.54 (m, 2H, $\left.\mathrm{C}^{\prime} 2-\mathrm{H}, \mathrm{C}^{\prime} 6-\mathrm{H}\right), 7.73(\mathrm{~s}, 1 \mathrm{H}, \mathrm{NH}), 9.18(\mathrm{~s}, 1 \mathrm{H}, \mathrm{NH}) ;{ }^{13} \mathrm{C} \mathrm{NMR}$ (100 MHz DMSO- $\left.d_{6}\right) \delta / p p m: ~ 14.1\left(\mathrm{CH}_{3}\right), 18.5\left(\mathrm{CH}_{3}-\mathrm{C} 6\right)$, $55.5\left(\mathrm{CH}_{2}\right), 59.9$ (C4), 101.2 (C5), 126.5 (C'4), 127.8 (C'2), 128.6 (C'3), 143.7 (C'1), 146.4 (C6), 153.7 (C2), 165.6 (C=0); Anal. Calcd. for $\mathrm{C}_{14} \mathrm{H}_{16} \mathrm{~N}_{2} \mathrm{O}_{3}$ : C, 64.60; $\mathrm{H}, 6.20 ; \mathrm{N}, 10.76$. Found: $\mathrm{C}, 64.41 ; \mathrm{H}, 6.32 ; \mathrm{N}, 10.59$.

Ethyl 1,2,3,4-tetrahydro-6-methyl-2-oxo-4-ptolylpyrimidine-5-carboxylate (4b):

White solid, (80\%); m.p. $=212-214 ;{ }^{1} \mathrm{H}$ NMR $(400 \mathrm{MHz}$, DMSO- $\left.d_{6}\right) \delta / p p m: 1.15\left(\mathrm{t}, J=7.2 \mathrm{~Hz}, 3 \mathrm{H}, \mathrm{CH}_{3} \mathrm{CH}_{2} \mathrm{O}\right), 2.3$ (s, $3 \mathrm{H}, \mathrm{CH} 3), 4.04\left(\mathrm{q}, J=7.2 \mathrm{~Hz}, 2 \mathrm{H}, \mathrm{CH}_{2}\right), 5.34(\mathrm{~s}, 1 \mathrm{H}, \mathrm{C} 4 \mathrm{H}), 6.22$ (s, 1H, NH), 7.05-7.16 (m, 4H, C'2-H, C'3-H, C'5-H, C'6-H), $8.75(\mathrm{~s}, 1 \mathrm{H}, \mathrm{NH}) ;{ }^{13} \mathrm{C}$ NMR (100 MHz DMSO-d $\left.d_{6}\right) \delta / \mathrm{ppm}$ : 14.1 $\left(\mathrm{CH}_{3}\right), 18.5\left(\mathrm{CH}_{3}-\mathrm{C} 6\right), 21.0\left(\mathrm{CH}_{3}-\mathrm{C}^{\prime} 4\right), 55.1\left(\mathrm{CH}_{2}\right), 59.8$ (C4), 101.2 (C5), 126.4 (C'4), 129.2 (C'2), 137.5 (C'3), 140.8 (C'1), 146.4 (C6), 153.9 (C2), 165.6 (C=O); Anal. Calcd. for $\mathrm{C}_{15} \mathrm{H}_{18} \mathrm{~N}_{2} \mathrm{O}_{3}: \mathrm{C}, 65.68 ; \mathrm{H}, 6.61 ; \mathrm{N}, 10.21$. Found: $\mathrm{C}, 65.85 ; \mathrm{H}$, 6.51; N, 10.38 .

Ethyl 1,2,3,4-tetrahydro-4-(2,3,4-trimethoxyphenyl)-6methyl-2-oxopyrimidine-5-carboxylate (4d):

White solid, (50 \%); m.p. $=195-197 ;{ }^{1} \mathrm{H}$ NMR $(400 \mathrm{MHz}$, DMSO- $\left.d_{6}\right) \delta / \mathrm{ppm}$ : $1.13\left(\mathrm{t}, 3 \mathrm{H}, J=7.2 \mathrm{~Hz}, \mathrm{CH}_{3} \mathrm{CH}_{2} \mathrm{O}\right), 2.43$ $\left(\mathrm{s}, 3 \mathrm{H}, \mathrm{CH}_{3}\right), 3.85\left(\mathrm{~s}, 3 \mathrm{H}, \mathrm{OCH}_{3}\right), 3.88\left(\mathrm{~s}, 3 \mathrm{H}, \mathrm{OCH}_{3}\right), 3.98$ (s, $\left.3 \mathrm{H}, \mathrm{OCH}_{3}\right), 4.08\left(\mathrm{q}, 2 \mathrm{H}, J=7.2 \mathrm{~Hz}, \mathrm{CH}_{2}\right), 5.54(\mathrm{~s}, 1 \mathrm{H}, \mathrm{C} 4 \mathrm{H})$, $6.58\left(\mathrm{~d}, 1 \mathrm{H}, J=8.8 \mathrm{~Hz}, \mathrm{C}^{\prime} 5 \mathrm{H}\right), 6.78\left(\mathrm{~d}, 1 \mathrm{H}, J=8.8 \mathrm{~Hz}, \mathrm{C}^{\prime} 6 \mathrm{H}\right)$, $7.30(\mathrm{~s}, 1 \mathrm{H}, \mathrm{NH}), 8.01(\mathrm{~s}, 1 \mathrm{H}, \mathrm{NH}) .{ }^{13} \mathrm{CNMR}(100 \mathrm{MHz}$, DMSO$\left.d_{6}\right) \delta / \mathrm{ppm}: 8.9\left(\mathrm{CH}_{3}\right), 13.3\left(\mathrm{CH}_{3}-\mathrm{C} 6\right), 44.8\left(\mathrm{CH}_{2}\right), 50.7(\mathrm{C} 4)$, $54.6\left(\mathrm{OCH}_{3}\right), 55.5\left(\mathrm{OCH}_{3}\right), 55.9\left(\mathrm{OCH}_{3}\right), 101.6(\mathrm{C} 5), 115.9$ (C'1), 122.7 ( $\left.C^{\prime} 6\right), 136.9$ (C'3), 142.6 ( $\left.C^{\prime} 4\right), 145.8$ ( $\left.C^{\prime} 2\right), 148.0$ (C6), 148.3 (C2), 160 (C=O); Anal. Calcd. for $\mathrm{C}_{17} \mathrm{H}_{22} \mathrm{~N}_{2} \mathrm{O}_{6}$ : C, 58.28; H, 6.33; N, 8.00. Found: C, 58.47; H, 6.43; N, 8.16.

Ethyl 4-(4-chlorophenyl)-1,2,3,4-tetrahydro-6-methyl-2oxopyrimidine-5-carboxylate (4e):

White solid, (60\%); m.p. $=211-213$; ${ }^{1 \mathrm{H}} \mathrm{NMR}(400 \mathrm{MHz}$, DMSO- $\left.d_{6}\right) \delta / p p m: 1.10\left(\mathrm{t}, 3 \mathrm{H}, J=7.2 \mathrm{~Hz}, \mathrm{CH}_{3} \mathrm{CH}_{2} \mathrm{O}\right), 2.25$ (s, $3 \mathrm{H}, \mathrm{CH}_{3}$ ), 3.98 (q, J = $7.2 \mathrm{~Hz}, 2 \mathrm{H}, \mathrm{CH}_{2}$ ), $5.14(\mathrm{~s}, 1 \mathrm{H}, \mathrm{C} 4 \mathrm{H})$, $7.25\left(\mathrm{~d}, 2 \mathrm{H}, J=8.4 \mathrm{~Hz}, \mathrm{C}^{\prime} 2-\mathrm{H}, \mathrm{C}^{\prime} 6 \mathrm{H}\right), 7.40(\mathrm{~d}, 2 \mathrm{H}, J=8.4 \mathrm{~Hz}$, $\left.\mathrm{C}^{\prime} 3-\mathrm{H}, \mathrm{C}^{\prime} 5 \mathrm{H}\right), 7.76(\mathrm{~s}, 1 \mathrm{H}, \mathrm{NH}), 9.24(\mathrm{~s}, 1 \mathrm{H}, \mathrm{NH}) ;{ }^{13} \mathrm{C} \mathrm{NMR}(100$ $\mathrm{MHz}$ DMSO- $\left.d_{6}\right) \quad \delta / \mathrm{ppm}: 14.1\left(\mathrm{CH}_{3}\right), 18.6\left(\mathrm{CH}_{3}-\mathrm{C} 6\right), 55.0$ $\left(\mathrm{CH}_{2}\right), 60.2$ (C4), 101.0 (C5), 127.9 (C'2), 128.8 (C'3), 133.7 (C'4), 142.1 ( $\left.C^{\prime} 1\right), 146.5$ (C6), 153.5(C2), 165.3 (C=O); Anal. Calcd. for $\mathrm{C}_{14} \mathrm{H}_{15} \mathrm{ClN}_{2} \mathrm{O}_{3}: \mathrm{C}, 57.05 ; \mathrm{H}, 5.13 ; \mathrm{N}, 9.50$. Found: $\mathrm{C}$, 57.22; H, 5.221; N, 9.66.

Ethyl 4-(2-chlorophenyl)-1,2,3,4-tetrahydro-6-methyl-2oxopyrimidine-5-carboxylate (4f):

White solid, (98\%); m.p. $=213-215 ;{ }^{1} \mathrm{H}$ NMR $(400 \mathrm{MHz}$, DMSO- $\left.d_{6}\right) \delta / p p m: 1.02\left(\mathrm{t}, 3 \mathrm{H}, \mathrm{CH}_{3} \mathrm{CH}_{2} \mathrm{O}\right) ; 2.45\left(\mathrm{~s}, 3 \mathrm{H}, \mathrm{CH}_{3}\right)$, $3.98\left(\mathrm{q}, J=7.2 \mathrm{~Hz}, 2 \mathrm{H}, \mathrm{CH}_{2}\right), 5.83(\mathrm{~s}, 1 \mathrm{H}, \mathrm{C} 4 \mathrm{H}), 5.95(\mathrm{~s}, 1 \mathrm{H}$, $\mathrm{NH})$, 7.20-7.35 (m, 4H, C'3-H, C'4-H, C'5-H, C'6-H), 9.02 (s, $1 \mathrm{H}, \mathrm{NH}) ;{ }^{13} \mathrm{C}$ NMR (100 MHz DMSO- $\left.d_{6}\right) \quad \delta / \mathrm{ppm}: 13.9$ $\left(\mathrm{CH}_{3}\right), 18.2\left(\mathrm{CH}_{3}-\mathrm{C} 6\right), 52.0\left(\mathrm{CH}_{2}\right), 59.9$ (C4), 98.8 (C5), 127.5 (C'5), 128.0 (C'3), 129.3 (C'4), 129.7(C'6), 132.4 (C'2), 139.6 (C'1), 148.6 (C6), 153.4 (C2), 165.4 (C=O); Anal. Calcd. for $\mathrm{C}_{14} \mathrm{H}_{15} \mathrm{ClN}_{2} \mathrm{O}_{3}: \mathrm{C}, 57.05 ; \mathrm{H}, 5.13 ; \mathrm{N}, 9.50$. Found: $\mathrm{C}, 57.24 ; \mathrm{H}$, 5.03; N, 9.67.

Ethyl 4-(4-formylphenyl)-1,2,3,4-tetrahydro-6-methyl-2oxopyrimidine-5-carboxylate (4h):

White solid, (97 \%); m.p. $=275-277 ;{ }^{1} \mathrm{H}$ NMR $(400 \mathrm{MHz}$, DMSO- $\left.d_{6}\right) \delta / p p m: 1.06\left(\mathrm{t}, 6 \mathrm{H}, J=6.9 \mathrm{~Hz}, 2 \mathrm{CH}_{3} \mathrm{CH}_{2} \mathrm{O}\right), 2.19$ $\left(\mathrm{s}, 6 \mathrm{H}, 2 \mathrm{CH}_{3}\right), 4.01\left(\mathrm{q}, 4 \mathrm{H}, J=6.9 \mathrm{~Hz}, 2 \mathrm{CH}_{2}\right), 5.14(\mathrm{~s}, 2 \mathrm{H}$, 2C4H), 7.19 (s, 4H, C'2-H, C'3-H, C'5-H, C'6-H), 9.60 (s, 2H, 2NH), 10.3 (s, 2H, 2NH); ${ }^{13} \mathrm{C}$ NMR (100 MHz DMSO-d $\left.d_{6}\right) \delta$ /ppm: $14.2\left(\mathrm{CH}_{3}\right), 18.5\left(\mathrm{CH}_{3}-\mathrm{C} 6\right), 55.1\left(\mathrm{CH}_{2}\right), 59.9(\mathrm{C} 4), 102.0$ (C5), 126.9 (C'2), 142.2 (C'1), 146.6 (C6), 152.5(C2), 166.3 (C=O); Anal. Calcd. for $\mathrm{C}_{22} \mathrm{H}_{26} \mathrm{~N}_{4} \mathrm{O}_{6}: \mathrm{C}, 59.72 ; \mathrm{H}, 5.92 ; \mathrm{N}$, 12.66. Found: $\mathrm{C}, 59.53 ; \mathrm{H}, 5.80 ; \mathrm{N}, 12.85$.

5-Acetyl-3,4-dihydro-6-methyl-4-p-tolylpyrimidin-2(1H)one $(4 \mathrm{p})$ :

White solid, (97\%); m.p. $=252-254 ;{ }^{1} \mathrm{H}$ NMR $(400 \mathrm{MHz}$, DMSO- $\left.d_{6}\right) \delta / p p m: 2.08$ (s, 3H, $\left.\mathrm{CH}_{3}-\mathrm{C} 6\right) .2 .25$ (s, 3H, $\mathrm{CH}_{3}-$ $\left.\mathrm{C}^{\prime} 4\right), 2.27\left(\mathrm{~s}, 3 \mathrm{H}, \mathrm{CH}_{3}-\mathrm{CO}\right), 5.22(\mathrm{~s}, 1 \mathrm{H}, \mathrm{C} 4 \mathrm{H}), 7.12-7.24(\mathrm{~m}$, $\left.4 \mathrm{H}, \mathrm{C}^{\prime} 2-\mathrm{H}, \mathrm{C}^{\prime} 3-\mathrm{H}, \mathrm{C}^{\prime} 5-\mathrm{H}, \mathrm{C}^{\prime} 6-\mathrm{H}\right), 7.75$ (s, 1H, NH), 9.13 (s, 1H, $\mathrm{NH}) ;{ }^{13} \mathrm{C}$ NMR (100 MHz DMSO-d 6 ) $\delta / \mathrm{ppm}: 19.1\left(\mathrm{CH}_{3}-\mathrm{C} 6\right)$, $21.5\left(\mathrm{CH}_{3}-\mathrm{C}^{\prime} 4\right), 31.2\left(\mathrm{CH}_{3}-\mathrm{CO}\right), 54.7$ (C4), 110.2 (C5), 127.4 (C'4), 130.2 (C'2), 137.5 (C'3), 142.8 (C'1), $149.4(C 6), 153.2$ (C2), 195.6 (C=O). Anal. Calcd. for $\mathrm{C}_{14} \mathrm{H}_{16} \mathrm{~N}_{2} \mathrm{O}_{2}: \mathrm{C}, 68.83 ; \mathrm{H}$, 6.60; N, 11.47. Found: C, 68.65; H, 6.71; N, 11.30 .

5-Aacetyl-3,4-dihydro-4-(2,3,4-trimethoxyphenyl)-6methylpyrimidin-2(1H)-one (4q):

White solid, (60\%); m.p. $=170-172 ;{ }^{1} \mathrm{H}$ NMR $(400 \mathrm{MHz}$, DMSO- $\left.d_{6}\right) \delta / p p m: 2.08\left(\mathrm{~s}, 3 \mathrm{H}, \mathrm{CH}_{3}\right), 2.41\left(\mathrm{~s}, 3 \mathrm{H}, \mathrm{CH}_{3}-\mathrm{CO}\right)$, $3.84\left(\mathrm{~s}, 3 \mathrm{H}, \mathrm{OCH}_{3}\right), 3.87\left(\mathrm{~s}, 3 \mathrm{H}, \mathrm{OCH}_{3}\right), 4.04\left(\mathrm{~s}, 3 \mathrm{H}, \mathrm{OCH}_{3}\right), 5.65$ (s, $1 \mathrm{H}, \mathrm{C} 4 \mathrm{H}), 6.57$ (d, $\left.1 \mathrm{H}, J=8.8 \mathrm{~Hz}, \mathrm{C}^{\prime} 5 \mathrm{H}\right), 6.75(\mathrm{~d}, 1 \mathrm{H}, J=8.8$ $\left.\mathrm{Hz}, \mathrm{C}^{\prime} 6 \mathrm{H}\right), 7.20$ (s, 1H, NH), 8.59 (s, 1H, NH). ${ }^{13} \mathrm{CNMR}(100$ $\left.\mathrm{MHz}, \mathrm{DMSO}-d_{6}\right) \delta / \mathrm{ppm}: \delta 14.1\left(\mathrm{CH}_{3}-\mathrm{C} 6\right), 24.3\left(\mathrm{CH}_{3}-\mathrm{CO}\right), 50.7$ (C4), $54.8\left(\mathrm{OCH}_{3}\right), 55.5\left(\mathrm{OCH}_{3}\right), 55.7\left(\mathrm{OCH}_{3}\right), 101.6(\mathrm{C} 5), 116.1$ (C'5), 119.8 (C'1), 121.8 (C'6) , 136.7 (C'3), 142.5 (C'4), 148.2 (C6), 148.7 (C2), $190.2(\mathrm{C}=\mathrm{O})$. Anal. Calcd. for $\mathrm{C}_{16} \mathrm{H}_{20} \mathrm{~N}_{2} \mathrm{O}_{5}: \mathrm{C}$, 59.99; H, 6.29; N, 8.74. Found: C, 60.19; H, 6.20; N, 8.92. 


\section{1-(1,2,3,4-Tetrahydro-6-methyl-2-thioxo-4-p- tolylpyrimidin-5-yl)ethanone (4v):}

White solid, (98\%); m.p. $=213-215 ;{ }^{1 H}$ NMR (400 MHz, DMSO- $\left.d_{6}\right) \delta / p p m: 2.11\left(\mathrm{~s}, 3 \mathrm{H}, \mathrm{CH}_{3}-\mathrm{C} 6\right), 2.30\left(\mathrm{~s}, 3 \mathrm{H}, \mathrm{CH}_{3}-\right.$ $\left.\mathrm{C}^{\prime} 4\right), 3.70\left(\mathrm{~s}, 3 \mathrm{H}, \mathrm{CH}_{3}-\mathrm{CO}\right), 5.24(\mathrm{~s}, 1 \mathrm{H}, \mathrm{C} 4 \mathrm{H}), 6.90-7.10(\mathrm{~m}$, $\left.4 \mathrm{H}, \mathrm{C}^{\prime} 2-\mathrm{H}, \mathrm{C}^{\prime} 3-\mathrm{H}, \mathrm{C}^{\prime} 5-\mathrm{H}, \mathrm{C}^{\prime} 6-\mathrm{H}\right), 9.68(\mathrm{~s}, 1 \mathrm{H}, \mathrm{NH}), 10.21(\mathrm{~s}, 1 \mathrm{H}$, $\mathrm{NH}) ;{ }^{13} \mathrm{C}$ NMR (100 MHz DMSO-d $\left.d_{6}\right) \delta / p p m: 19.1\left(\mathrm{CH}_{3}-\mathrm{C} 6\right)$, $21.2\left(\mathrm{CH}_{3}-\mathrm{C}^{\prime} 4\right), 31.4\left(\mathrm{CH}_{3}-\mathrm{CO}\right), 56.7$ (C4), 111.2 (C5), 127.3 (C'4), 128.3 (C'2), 136.0 (C'3), 145.4 (C'1), 159.1 (C6), 174.2 (C2), 195.6 (C=S); Anal. Calcd. for $\mathrm{C}_{14} \mathrm{H}_{16} \mathrm{~N}_{2} \mathrm{OS}: \mathrm{C}, 64.58 ; \mathrm{H}$, 6.19; N, 10.76; S, 12.32. Found: C, 64.77; H, 6.11; N, 10.94; $\mathrm{S}, 12.48$.

1-(4-(2-Chlorophenyl)-1,2,3,4-tetrahydro-6-methyl-2thioxopyrimidin-5-yl)ethanone (4x):

White solid, (98 \%); m.p. $=175-177 ;{ }^{1} \mathrm{H}$ NMR $(400 \mathrm{MHz}$, DMSO- $\left.d_{6}\right) \delta / \mathrm{ppm}: 2.07$ (s, $\left.\mathrm{CH}_{3}-\mathrm{C} 6\right), 2.32\left(\mathrm{~s}, 3 \mathrm{H}, \mathrm{CH}_{3}-\mathrm{CO}\right)$, 5.65 (s, 1H, C4H), 7.18-7.33 (m, 3H, C'4-H, C'5-H, C'6-H), 7.35-7.37 (m, 1H, ' ' $3-\mathrm{H}), 9.60(\mathrm{~s}, 1 \mathrm{H}, \mathrm{NH}), 10.30(\mathrm{~s}, 1 \mathrm{H}, \mathrm{NH})$; ${ }^{13} \mathrm{C}$ NMR (100 MHz DMSO- $\left.d_{6}\right) \delta / p p m: 18.6\left(\mathrm{CH}_{3}-\mathrm{C} 6\right), 30.7$ ( $\left.\mathrm{CH}_{3}-\mathrm{CO}\right), 52.3$ (C4), 110.1 (C5), 128.5 (C'5), 129.0 (C'3), 130.1 (C'4), 132.3 (C'6), 140.1 (C'1), 145.3 (C6), 174.4 (C2), 195.2 (C=S); Anal. Calcd. for $\mathrm{C}_{13} \mathrm{H}_{13} \mathrm{ClN}_{2} \mathrm{OS}$ : C, 55.61; H, 4.67; $\mathrm{N}, 9.98 ; \mathrm{S}, 11.42$. Found: $\mathrm{C}, 55.42 ; \mathrm{H}, 4.75 ; \mathrm{N}, 9.81 ; \mathrm{S}, 11.59$.

Acknowledgment. We express our thanks to the Research Council of Shahrood University of Technology for the financial support of this work.

\section{REFERENCES}

[1] G. C. Rovnyak, S. D. Kimball, B. Beyer, G. Cucinotta, J. D. Dimarco, J. Gougoutas, A. Hedberg, M. Malley, J. P. McCarthy, R. Zhang, S. Moreland, J. Med. Chem. 1995, 38, 119.

[2] C. O. Kappe, W. M. F. Fabian, M. A. Semones, Tetrahedron 1997, 53, 2803

[3] R. C. Rizzo, J. Tirado-Rives, W. L. Jorgensen, J. Med. Chem. 2001, 44, 145.

[4] K. Barral, J. Courcambeck, G. Pepe, J. Balzarini, J. Neyts, E. D. Clercq, M. Camplo, J. Med. Chem. 2005, 48, 450.

[5] P. Biginelli, Gazz. Chim. Ital. 1893, 23, 360.

[6] K. Folkers, T. B. Johnson, J. Am. Chem. Soc. 1933, 55, 2886.

[7] E. H. Hu, D. R. Sidler, H. U. Dolling, J. Org. Chem. 1998, 63, 3454.

[8] V. Radharani, N. Srinivas, M. Radha Kishan, S. J. Kulkarni, K. V. Raghavan, Green Chem. 2001, 3, 305.

[9] W. Su, J. Li, Z. Zheng, Y. Shen, Tetrahedron Lett. 2005, 46, 6037.

[10] A. R. Gholap, K. Venketasan, T. Daniel, R. J. Lahoti, K. V. Srinivasan, Green Chem. 2004, 6, 147.
[11] X. Zhang, Y. Li, C. Liu, J. J. Wang, J. Mol. Catal. A: Chem. 2006, 253, 207.

[12] K. R. Reddy, C. V. Reddy, M. Mahesh, P. V. K. Raju, V. V. N. Reddy, Tetrahedron Lett. 2003, 44, 8173.

[13] Y. Zhang, B. Wang, X. Zang, J. Huang, C. Liu, Molecules 2015, 20, 3811.

[14] J. Pavlinac, M. Zupan, K. K. Laali, S. Stavber, Tetrahedron 2009, 65, 5625.

[15] P. Hapiot, C. Lagrost, Chem. Rev. 2008, 108, 2238.

[16] P. P. Salvi, A. M. Mandhare, A. S. Sartape, D. K. Pawar, S. H. Han, S. S. Kolekar, C. R. Chim. 2011, 14, 883.

[17] N. J. Ghaffari Khaligh, J. Mol. Catal. A Chem. 2011, 34, 63.

[18] A. S. Amarasekara, Chem. Rev. 116 (2016) 6133-6183.

[19] M. Bakherad, A. Keivanloo, S. Samangooei, Tetrahedron Lett. 2012, 53, 5773.

[20] M. Bakherad, A. Keivanloo, S. Jajarmi, Tetrahedron 2012, 68, 2107.

[21] A. Keivanloo, M. Mirzaee, M. Bakherad, A. Soozani, Chin. J. Catal. 2014, 35, 362.

[22] R. Tayebee, M. Jomei, B. Maleki, M. Kargar Razi, H. Veisi, M. Bakherad, J. Mol. Liq. 2015, 206, 119.

[23] M. N. Esfahani, M. Montazerozohori, M. A. Mirrezaee, H. Kashi, J. Chil. Chem. Soc. 2014, 59, 2311.

[24] S. K. Pardeshi, D. D. Kumbhar, B. Y. Waghamare, P. D. Lokhande, J. Pharm. Biol. Chem. Sci. 2013, 4, 1117.

[25] E. Kolvari, N. Koukabi, O. A. Armandpour, Tetrahedron 2014, 70, 1383.

[26] S. V. Shinde, W. N. Jadhav, M. K. Lande, L. S. Gadekar, B. R. Arbad, J. M. Kondre, N. Karadeet, Catal. Lett. 2008, 125, 57.

[27] H. Eshghi, A. Javid, A. Khojastehnezhad, F. Moeinpour, F. F. Bamoharram, M. Bakavoli, M. Mirzaei, Chin. J. Catal. 2015, 36, 299.

[28] D. An, Y.-S. Fan, Y. Gao, Z.-Q. Zhu, L.-Y. Zheng, S.-Q. Zhang, Eur. J. Org. Chem. 2014, 301.

[29] J. C. Rodriguez-Domi, D. Bernardi, G. Kirsch, Tetrahedron Lett. 2007, 48, 5777.

[30] E. Rafiee, H. A. Jafari, Bioorg. Med. Chem. Lett. 2006 , 16, 2463.

[31] E. Rafiee, F. Shahbazi, J. Mol. Catal. A: Chem. 2006, 250, 57.

[32] S. P. Maradur, G. S. Gokavi, Catal. Commun. 2007, 8, 279.

[33] K. Sujatha, P. Shanmugam, P. T. Perumal, D. Muralidharan, M. Rajendran, Bioorg. Med. Chem. Lett. 2006, 16, 4893.

[34] C. Mukhopadhyay, A. Datta, B. K. Banik, J. Heterocycl. Chem. 2007, 44, 979. 